\title{
Strain-driven self-rolling of hybrid organic-inorganic microrolls: interfaces with self-assembled particles
}

\author{
Masahide Takahashi ${ }^{1}$, Cristiana Figus ${ }^{2}$, Luca Malfatti ${ }^{2}$, Yomei Tokuda ${ }^{3}$, Kazuyuki Yamamoto ${ }^{3}$, \\ Toshinobu Yoko ${ }^{3}$, Takuya Kitanaga ${ }^{1}$, Yasuaki Tokudome ${ }^{1}$ and Plinio Innocenzi ${ }^{2}$ \\ Strain driven micro and nanoroll fabrication is generally restricted to multilayer and multiprocessing systems, limiting the \\ ability to exploit self-organization at different length scales. We have designed a hybrid organic-inorganic single-layered film \\ with a surface that responds selectively to external stimuli, resulting in mechanical strain and self-rolling in one-step \\ fabrication. The scrolling is initiated by water and any aqueous solution of molecules or colloidal particles. During scrolling, the \\ different species in solution remain entrapped in the rolls; the constrained environment at the interface of the roll walls pushes \\ the particles to organize into ordered structures. We used this rolling process to create self-assembled hybrid films with \\ well-ordered layers of gold nanoparticles and opals of polystyrene nanospheres. These films also respond selectively to solvents, \\ allowing the easy release of molecules/particles entrapped at the interface.
}

NPG Asia Materials (2012) 4, e22; doi:10.1038/am.2012.40; published online 20 July 2012

Keywords: self-assembly; microrolls; nanoparticle; smart materials; sol-gel; hybrid organic-inorganic materials

\section{INTRODUCTION}

The strain-driven self-rolling of films is an advanced engineering strategy for fabricating micro- and nanotubes of different sizes and compositions. ${ }^{1}$ Self-rolling is activated when the surface and bottom layers of a film show a distinctive mechanical response upon the application of specific conditions that induce a strain. Self-rolling is a common natural phenomenon observed in leaves and clays, and the challenge in materials science is to master the process sufficiently to achieve controlled rolling. Mechanical stress arises from structural heterogeneities in the film, ${ }^{2}$ such as chemical inhomogeneities due to a gradient in composition, ${ }^{3-5}$ differences in the lattice constants between the top and bottom layers, ${ }^{6,7}$ or differences in the degree of crosslinking in the case of polymers. Detachment of the film with release of the strain causes scrolling of the layer and the formation of tubes and rolls; this mechanical response is quite general and has been observed in various materials such as metals, ${ }^{8}$ semiconductors, ${ }^{9,10}$ polymers ${ }^{11,12}$ and oxides. ${ }^{13}$ The main strategy that has been employed thus far is to induce the stress by fabricating bilayer or multilayer films, in which the mismatch between the two layers is used as the bending driving force. In a multilayer structure the bottom film acts as sacrificial layer and is selectively etched. ${ }^{14}$ In the case of polymeric materials, the bottom film exhibits a selective swelling response that also induces mechanical stress. ${ }^{4}$ Multilayer configurations are employed for inducing self-scrolling even for organic polymers, in general via selective swelling ${ }^{15}$ or thermal expansion mismatch. ${ }^{16,17}$ In the case of metal or semiconductor films, the curvature of the bending layer depends on the mechanical strain, stiffness and film thickness, ${ }^{2}$ whereas in organic polymeric layers the mechanical response is also affected by the degree of crosslinking, swelling in selective solvents and viscoelastic properties. Although several examples of self-rolling have been reported in the literature with different types of materials, these cases are strictly limited to bilayer or multilayered materials because inducing a mechanical misfit that triggers the driving force for bending is extremely difficult in other materials.

This work presents an alternative approach to obtaining microrolls of a thin organic-inorganic hybrid layer that does not require any multilayer structure. In addition to its potential simplicity and versatility, this method also presents some distinctive advantages for the fabrication of various advanced functional devices. Furthermore, the much simpler single step fabrication route could allow the integration of self-rolling microroll formation with the processing and ordering of micro and nanoparticles at the wall interfaces. This implementation of the process would be particularly attractive

\footnotetext{
${ }^{1}$ Department of Materials Science, Osaka Prefecture University, Sakai, Osaka, Japan; ${ }^{2}$ Laboratorio di Scienza dei Materiali e Nanotecnologie, CNSB, CR-INSTM, Università di Sassari, Alghero, Italy and ${ }^{3}$ Institute for Chemical Research, Kyoto University, Uji, Kyoto, Japan

Correspondence: Professor M Takahashi, Department of Materials Science, Osaka Prefecture University, Sakai, Osaka 599-8531, Japan

E-mail: masa@photomater.com

or Professor Y Tokuda, Institute for Chemical Research, Kyoto University, Uji, Kyoto 611-0011, Japan.

E-mail: tokuda@noncry.kuicr.kyoto-u.ac.jp

or Professor P Innocenzi, Laboratorio di Scienza dei Materiali e Nanotecnologie, CNSB, CR-INSTM, Università di Sassari, Palazzo Pou Salit, Piazza Duomo 6, Alghero (SS) 07041, Italy.

E-mail: plinio@uniss.it

Received 23 February 2012; revised 6 June 2012; accepted 6 June 2012
} 
because a single step could produce a roll that contains functional or sensing layers incorporated in a closed environment with the molecules to be analyzed.

\section{MATERIALS AND METHODS}

The chemicals 3-glycidoxypropyltrimethoxysilane (GPTMS, 98\%, SigmaAldrich, St Louis, MO, USA), sodium hydroxide $(\mathrm{NaOH}$, pellets $98 \%$, Carlo Erba, Milan, Italy) and hydrochloroauric acid tetrahydrate $\left(\mathrm{HAuCl}_{4} \cdot 4 \mathrm{H}_{2} \mathrm{O}\right.$, Aldrich) were used as received without further purification.

\section{Preparation of precursor film and microrolls}

The precursor sol was prepared by the addition of GPTMS $(10 \mathrm{ml})$ to an aqueous $\mathrm{NaOH}$ solution $(4 \mathrm{ml}, 1.85 \mathrm{M}, \mathrm{pH} 14)$ with stirring. The molar ratio of the components was set to GPTMS: $\mathrm{H}_{2} \mathrm{O}: \mathrm{NaOH}=1: 5: 0.167$. Immediately after the preparation, the precursor sol was left in an open vessel at $25^{\circ} \mathrm{C}$ and $40 \%$ relative humidity for $30 \mathrm{~min}$ to allow the evaporation of methanol, a byproduct of the sol-gel reactions. After this time, the container was sealed, and the sol was left to react, along with stirring for $12 \mathrm{~h}$ at $25^{\circ} \mathrm{C}$. At the end of this first preparation step, the sol was aged in a closed vessel for 6 days at $25^{\circ} \mathrm{C}$; hybrid films from the aged sol were deposited by spin-coating on silicon substrates at a rotation speed of 3000 r.p.m. for 20 s. The substrates were cleaned with water and acetone and rinsed with ethanol prior to use. After deposition, the films were dried in an oven at $90^{\circ} \mathrm{C}$ for $20 \mathrm{~min}$ and immersed in water to induce self-rolling.

\section{Dye-doped rolls}

Microrolls containing Rhodamine 6G (Rh6G) were prepared in two different ways: one-pot synthesis, through direct dissolution into the precursor sol, or interfacial entrapment during the self-rolling process. In the first case, Rh6G was added to the precursor solution to reach a molar ratio of GPTMS:Rh6G $=1: 5 \times 10^{-4}$. In the second case, an aqueous Rh6G solution $\left(1.5 \times 10^{-3} \mathrm{~mol} / \mathrm{l}\right)$ was poured on the hybrid film surface to induce scrolling.

\section{In-situ nucleation of gold nanoparticles}

An aqueous solution containing the gold precursor was prepared by mixing $0.05 \mathrm{~g}$ of $\mathrm{HAuCl}_{4} \cdot 4 \mathrm{H}_{2} \mathrm{O}$ with $4 \mathrm{ml}$ of water. The solution was used for scrolling of the films by casting a few droplets on their surfaces; after formation, the rolls were exposed to ultraviolet (UV) light for different periods of time, up to $30 \mathrm{~min}$. A 500-W super-high-pressure mercury arc lamp equipped with a band pass filter of $365 \mathrm{~nm}$ (UVC-502, SAN-EI Electric, Japan) was used as the excitation source. The power density was measured as $470 \mu \mathrm{W} \mathrm{cm}{ }^{-2}$ at the sample surface (accumulated irradiance: $846 \mathrm{~J} \mathrm{~cm}^{-2}$ ).

In the case of roll fabrication with co-doped Rh6G and gold nanoparticles, the aqueous Rh6G solution and the gold precursor solution were mixed in the ratio $1: 1$ and applied to induce strain.

\section{Wrapping of ceria NPs}

Rolls were also obtained with ceria nanoparticles at the interface by dipping the films after thermal treatment in a solution of ceria nanoparticles in water. The ceria nanoparticles were synthesized by adding a solution of cerium nitrate $(0.7 \mathrm{~g})$ and 2-propanol $(2 \mathrm{ml})$ to a solution of cetyltrimethyl ammonium bromide (CTAB $0.2 \mathrm{~g})$ and 2-propanol $(2 \mathrm{ml})$.

\section{Polystyrene particles at the roll interface}

An aqueous solution of $240 \mathrm{~nm}$ diameter polystyrene nanoparticles at $10 \% \mathrm{vo}$ was poured on the film surface to induce scrolling and allow for the entrapment of the particles at the interfaces of the roll layers.

\section{Experimental procedures}

UV-visible (UV-Vis) spectra were recorded on a Thermo Nicolet Evolution 300 UV-visible spectrophotometer (Thermo Nicolet, Schwerte, Germany).

$\mathrm{X}$-ray diffraction patterns were collected in the angular range from $1^{\circ}$ to $70^{\circ}$, using a Bruker Discovery-8 instrument (Bruker AXS GmbH, Karlsruhe, Germany) in the Bragg-Brentano geometry with a copper tube $(\lambda=1.54056 \AA)$; the X-ray generator was set to a power of $40 \mathrm{kV}$ and $40 \mathrm{~mA}$
Films deposited on silicon substrates were subjected to infrared absorption analysis with a Bruker Vertex70 FTIR spectrometer (Bruker Optik GmbH, Ettingen, Germany) in transmission mode in the range $400-4000 \mathrm{~cm}^{-1}$; the data were collected over 256 scans with a resolution of $4 \mathrm{~cm}^{-1}$ and silicon was used as the background.

A Bruker Senterra confocal Raman microscope (Bruker Optik GmbH) with a laser excitation wavelength of $532 \mathrm{~nm}$ at increasing nominal power and a $\times 20$ objective was used for surface enhanced raman scattering (SERS) measurements. Each spectrum was obtained with the same focal conditions by averaging five acquisitions of $5 \mathrm{~s}$ each with a resolution range of $9 \mathrm{~cm}^{-1}$.

The morphology of the sample was investigated by a scanning electron microscope (SEM, S-4800, Hitachi, Tokyo, Japan) and a transmission electron microscope (TEM, JEM-2000FX, JEOL, Tokyo, Japan) operated at $200 \mathrm{kV}$. The scanning electron microscope was operated at $10 \mathrm{kV}$ acceleration voltage and $10 \mu \mathrm{A}$ current.

Fluorescence analysis was performed using a FluoroMax-3 Horiba JobinYvon spectrofluorometer (Jobin-Yvon, Longjumeau, France). The acquisitions are the averages of three different accumulations; emission spectra were collected between 500 and $700 \mathrm{~nm}$ using an excitation wavelength of $480 \mathrm{~nm}$.

\section{RESULTS}

\section{Formation of rolls via strain-driven self-scrolling}

A hybrid organic-inorganic film of GPTMS, an organically modified alkoxide bearing an epoxy, ${ }^{18}$ was obtained by sol-gel synthesis in highly basic conditions and used as the precursor layer for the preparation of microrolls. This hybrid coating is optically transparent, and its thickness can be adjusted over a large range, typically from $0.3 \mu \mathrm{m}$ to hundreds of $\mu \mathrm{m}$, by controlling the processing parameters such as the spin coating rate. The high $\mathrm{pH}$ in the precursor sol allows a rapid rate of hydrolysis that slows down at the same time as the condensation reactions. Therefore, after deposition, the material remains in a softlike state, and thermal treatment at $90^{\circ} \mathrm{C}$ is enough to produce evaporation of the residual solvent and partial polycondensation of the silica network. When the film comes in contact with water, it is detached from the substrate and scrolls to form microrolls; the rolls remain optically transparent and exhibit a multiwall structure (Figure 1). The process is highly reproducible, and it is possible to control roll diameter, dimensions and the number of roll walls by adjusting the processing parameters such as the composition, time and temperature of heat treatment, and surface properties of the substrate (Supplementary Information 1). The mechanism of formation of the microroll is shown in Figure 2 and will be described later in Discussion.

\section{Incorporation of functional species within the roll walls}

Sol-gel processing of hybrid films allows the integration of different functional molecules and particles into the roll walls through one-pot synthesis; one example is the incorporation of the fluorescent dye Rh6G within the hybrid film by direct introduction of the dye into the precursor sol. After scrolling, the microroll walls contain the fluorescent Rh6G, as shown by optical absorption and fluorescence spectra (Figure 3 ) that exhibit the typical signature of the dye. ${ }^{19}$

\section{In-situ formation of gold nanoparticles at the interface}

This method of fabricating nanocomposite hybrids that self-assemble in a specific shape is flexible enough to allow for the entrapment of functional species not only in the roll wall, as just described, but also at the interfaces between adjacent walls. To accomplish this, $\mathrm{HAuCl}_{4} \cdot \mathrm{H}_{2} \mathrm{O}$ was dissolved as the gold precursor in water and this aqueous solution was used to induce mechanical strain in the films; the microrolls were then exposed to UV light to form gold nanoparticles and the exposure time was used to tune the particle dimensions. Interestingly, at long exposure times of several hours, the 

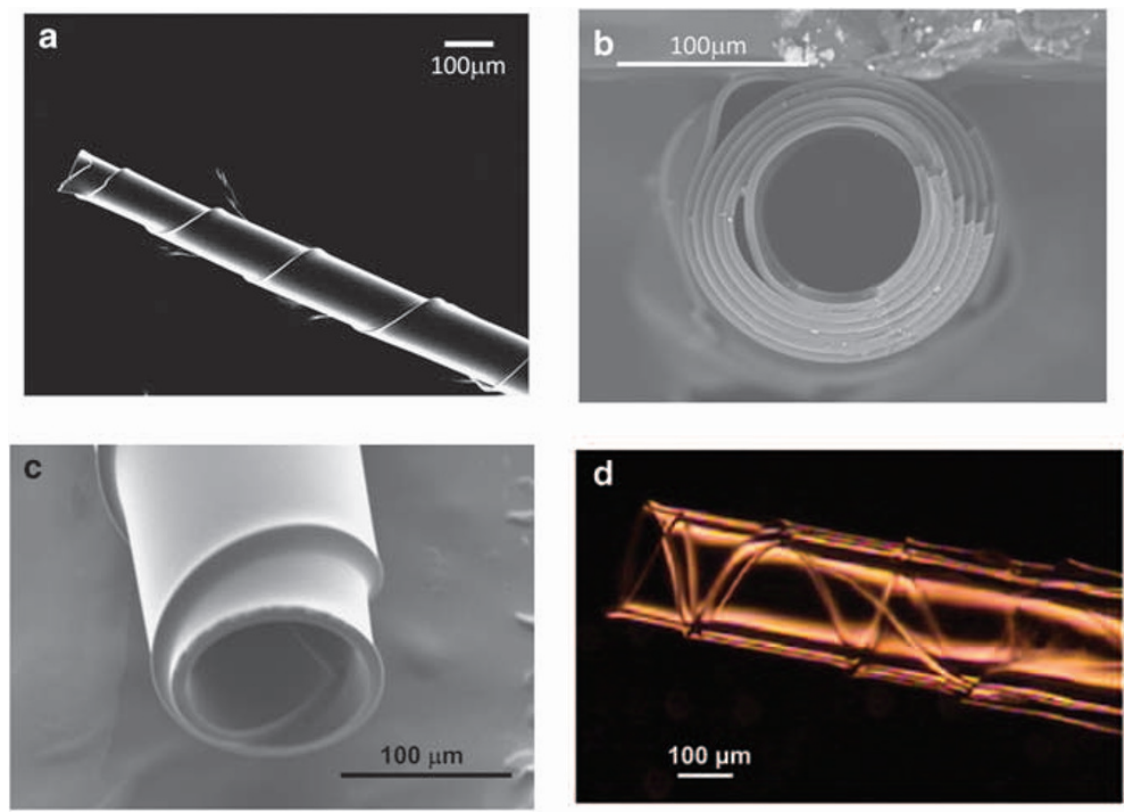

Figure 1 Scanning electron microscope $(\mathbf{a}-\mathbf{c})$ and optical (d) images of the microrolls reveal a multi-layer tubular structure obtained by self-wrapping of a pristine hybrid film.

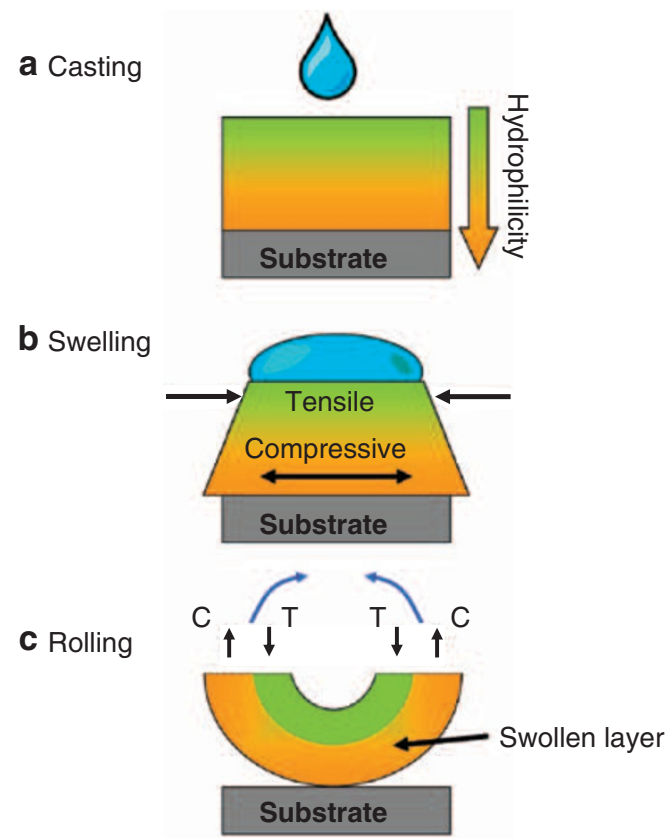

Figure 2 This scheme depicts the three steps of self-rolling. A water droplet is cast over a hybrid film after thermal treatment (a). The different degrees of condensation between the upper and lower parts of the film induces a gradient of hydrophilicity from the top to the bottom of the layer (b). A tensile stress $(T)$ arises in the upper part of the film and a compressive stress (C) in the bottom part. Water preferentially swells the inner part of the film, causing swelling and an increase in the mechanical stress; once the stress reaches a threshold, the film comes off from the substrate and the strain induces rolling up (c).

coalescence of the particles leads to the formation of a gold layer at the interface (Supplementary Information 2), indicating that the gold precursor is continuously supplied from the aqueous solution by capillarity. The formation of gold nanoparticles is confirmed by

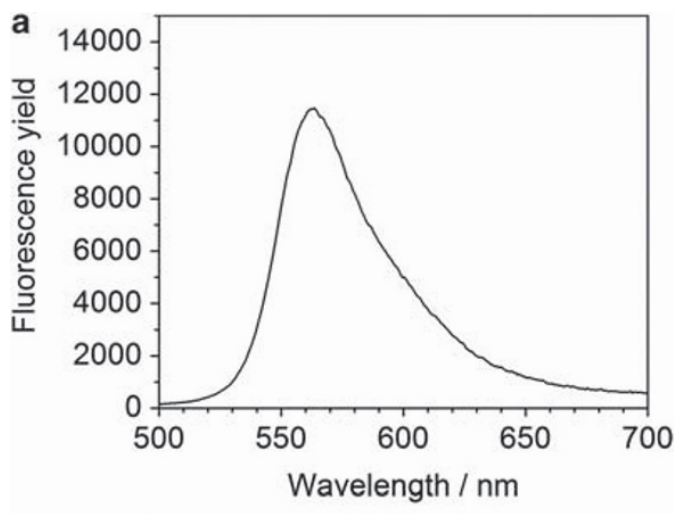

b

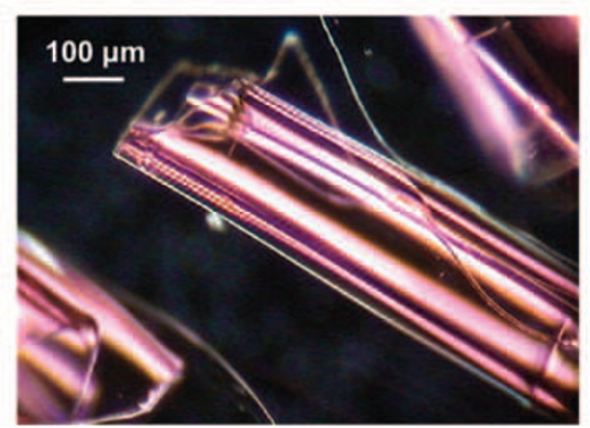

Figure 3 Functional properties such as fluorescence or non-linear optical emission can be induced in the microrolls if a fluorescent dye is dissolved in the initial sol. (a) Emission spectrum of microrolls deposited on a silica glass slide and (b) optical image of a fluorescent microroll upon illumination with a halogen lamp.

UV-Vis absorption spectra, X-ray diffraction and electron diffraction imaging (Figure 4), and a schematic of the rolls wrapped with gold nanoparticles is shown in Figure 5a. A scanning electron microscope image of a cross-section of the roll also confirms that the gold 

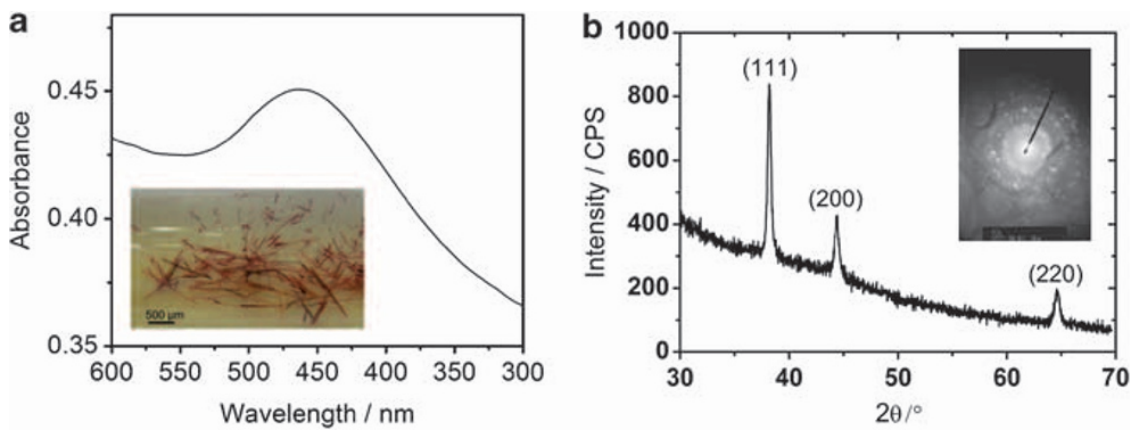

Figure 4 UV-Vis spectrum showing the plasmonic absorption band of rolls that contain gold nanoparticles at the wall interfaces; the inset shows an optical image of the microrolls (a). X-ray diffraction patterns of rolls containing gold nanoparticles, where the diffraction peaks are due to the formation of metallic gold; the inset shows the electron diffraction pattern of the particles at the interface, which is also assigned to gold (b).
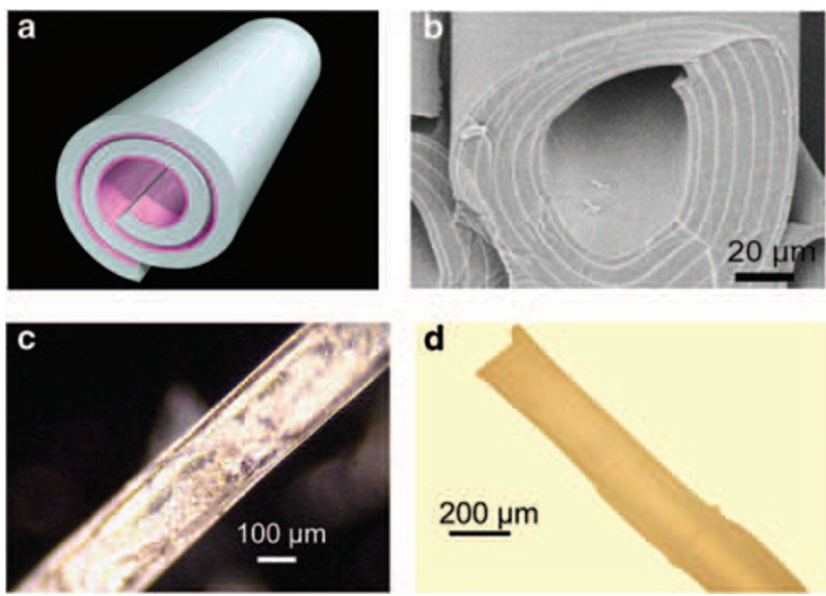

Figure 5 Entrapment of nanoparticles formed in situ and ex situ. A schematic depicting entrapment of functional species at the interface of adjacent walls (a). Gold nanoparticles are formed in situ by an integrated process: the gold precursor $\left(\mathrm{HAuCl}_{4} \cdot 4 \mathrm{H}_{2} \mathrm{O}\right)$ is dissolved in the aqueous solution used for the rolling up. After UV illumination, a well-ordered layer of gold nanoparticles is formed among the roll coils, as shown in the SEM image of a section of the roll containing the layer of gold nanoparticles at the wall interfaces. The gold layers appear in white in the image (b). Ceria (c) and PS nanoparticles (d), which were formed ex situ and dissolved in an aqueous solution, are also entrapped during self-rolling; the organization of PS particles at the interface makes the rolls opalescent.

nanoparticle layers, which appear white in the image, are formed at the wall interfaces (Figure 5b).

\section{Incorporation of functional species at the interface}

We have also extended this formation procedure to other types of functional nanoparticles prepared $e x s i t u$, such as $\mathrm{CeO}_{2}$ (Figure $5 \mathrm{c}$ and Supplementary Information 3), super paramagnetic $\mathrm{Fe}_{2} \mathrm{O}_{3}$ magnetite and mesoporous silica nanoparticles. In addition to nanoparticles, we have also tested the ability of this method to entrap monodisperse microparticles fabricated ex situ. Water droplets containing monodisperse polystyrene (PS) particles with an average diameter of 240 $\mathrm{nm}$ were poured on the surface of the hybrid film to induce scrolling. The PS particles remain well entrapped at the wall interface, leading the microrolls to appear opalescent (Figure $5 \mathrm{~d}$ ).
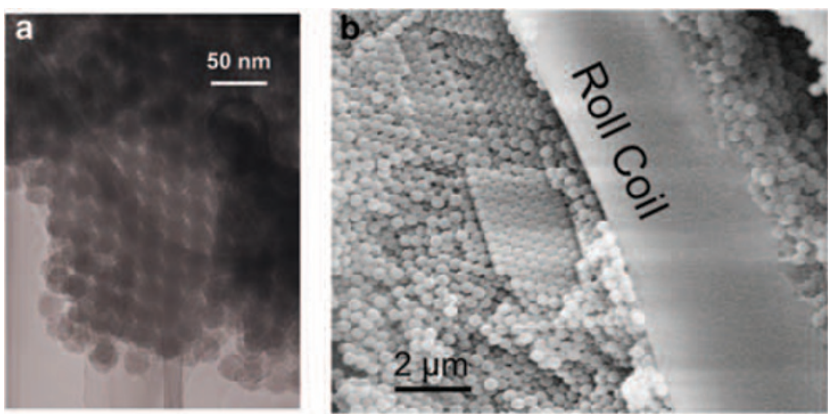

Figure 6 The gold nanoparticles that form in situ at the interface selfassemble into a well-ordered 3D array (a). Photonic crystals prepared through self rolling triggered by an aqueous colloidal solution of polystyrene nanospheres (average dimension $\approx 240 \mathrm{~nm}$ ) (b).

\section{Self-assembly at the interface}

Assembly of the nano- and microparticles into ordered arrays at the interface has been observed. The transmission electron microscope image of gold nanoparticles embedded in the microroll shown in Figure 4 shows that the gold nanoparticles have a uniform average dimension of $30 \mathrm{~nm}$ and that they self-order into a 3D array at the interface (Figure 6a). On the other hand, the cross-sectional scanning electron microscope image of the microroll prepared with PS microparticles in Figure 5d shows that the monodisperse PS nanoparticles captured during the scrolling process form well-packed arrays between adjacent roll coils with highly ordered domains (Figure 6b). The process of entrapping the nanoparticles at the interface is illustrated in Figure 7, which reproduces the microroll wrapping and the ordering of nano- and microparticles. A colloidal solution containing the particles is used to trigger the rolling-up process on a flat hybrid film (Figure 7a); rolling takes place after the aqueous solution is cast (Figure $7 \mathrm{~b}$ ), and then particles self-assemble by steric hindrance (Figure $7 \mathrm{c}$ ).

\section{Multifunctional rolls for sensing applications}

Multifunctional microrolls have been fabricated as sensing devices and used as a proof of concept of the different possibilities offered by this versatile method. Microrolls containing both Rh6G and gold nanoparticles were prepared; an aqueous solution containing Rh6G and the gold precursor was used to induce the mechanical strain in the hybrid coating, leading to the entrapment of these two species at the interface after the scrolling process. UV exposure of the rolls induces 

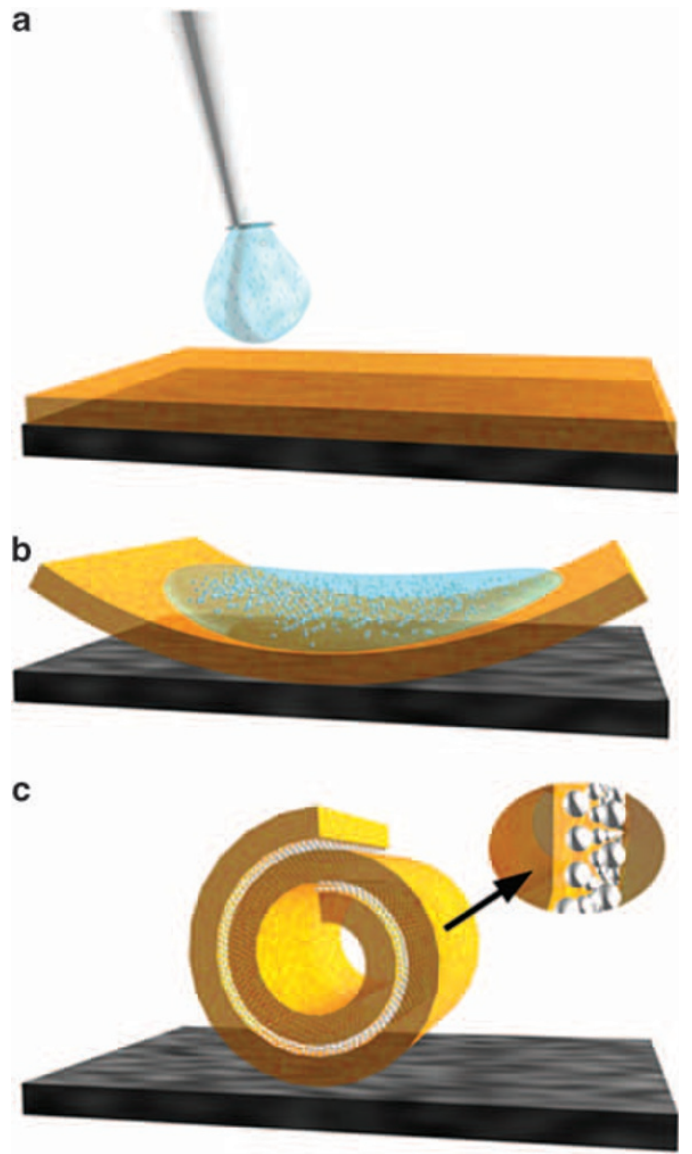

Figure 7 These drawings depict the microroll wrapping and ordering of nanoand microparticles. A colloidal solution containing the particles is used to trigger the rolling-up process on a flat hybrid film (a). After deposition, rolling occurs (b) and particles self-assemble due to steric hindrance (c).

the formation of gold nanoparticles that are in direct contact with Rh6G. The UV-Vis absorption spectra of the microrolls (Figure 8a) show two overlapping bands, one with a peak at $500 \mathrm{~nm}$ assigned to the plasmonic peak of gold nanoparticles and another at $540 \mathrm{~nm}$ due to Rh6G. This system represents a SERS system in which the gold nanoparticles come into contact with the analyte Rh6G during the UV illumination; this process also has the advantage that the sensing molecules are concentrated at the interface by capillarity during the self-rolling. Raman spectra of a microroll doped with Rh6G at the interface and co-doped with gold nanoparticles and Rh6G are shown in Figure $8 \mathrm{~b}$. The sample containing both gold nanoparticles and Rh6G shows enhanced Raman scattering, taking into account the different laser power; the amplification of the Raman signal is significant considering that the microroll formation process has not been optimized for SERS measurements.

\section{DISCUSSION}

Hybrid organic-inorganic materials are an interesting option for preparing microrolls because they exhibit properties intermediate between those of organic polymers and inorganic oxides. These materials are prepared via low temperature sol-gel chemistry, which allows wide flexibility in terms of composition and properties and has the advantage that functional molecules or nanoparticles can be easily incorporated within the material by one-pot synthesis. ${ }^{20}$ Hybrid films are generally amorphous in structure, although it has very recently
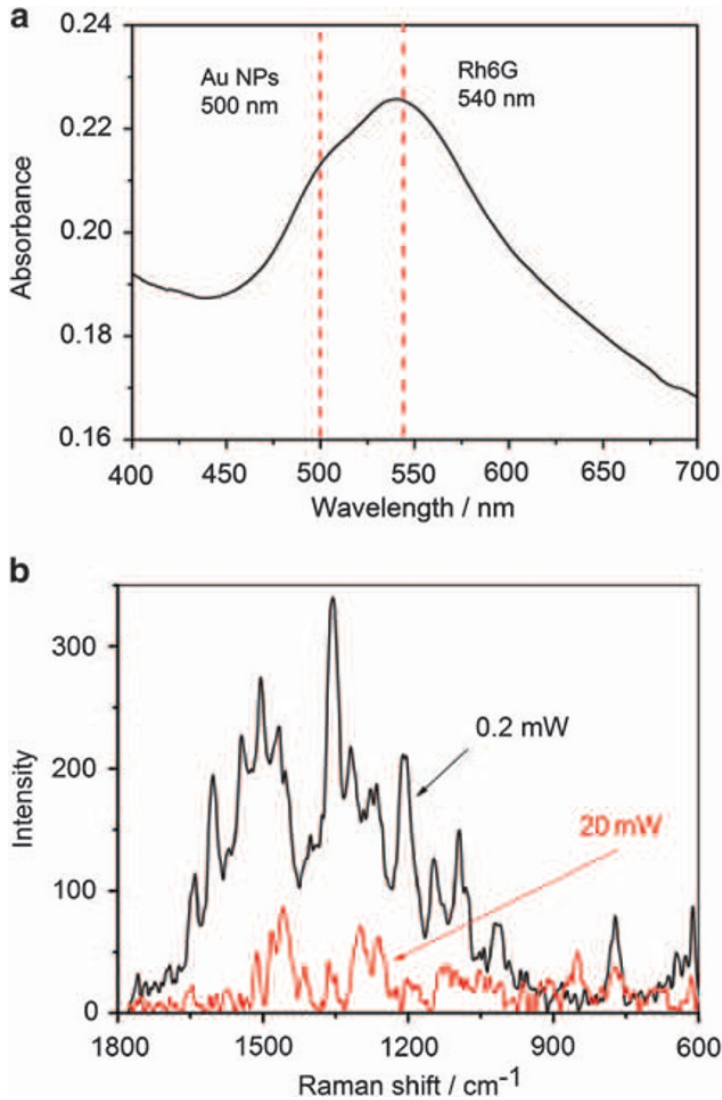

Figure 8 UV-Vis absorption spectrum of microrolls containing both Rh6G and gold nanoparticles (a). The spectra show the typical plasmonic peak of gold nanoparticles, $\sim 500 \mathrm{~nm}$, which overlaps with the Rh6G absorption band approximately $540 \mathrm{~nm}$. Raman spectra of a microroll doped with Rh6G at the interface (red line) and gold nanoparticles and Rh6G (black line) (b).

been demonstrated for the GPTMS system that lamellar nano crystalline-containing hybrid films can also be obtained under specific conditions. ${ }^{21,22}$ The particular organic-inorganic material employed here allows formation of hybrid crystalline films containing ordered layered structures, enabling control of the physical properties of the wall material itself (Supplementary Information 4 and 5).

The strain-driven scrolling mechanism is quite simple to explain. After heat treatment, the bottom part of the film remains less condensed and more hydrophilic, and swelling with water produces the scrolling of the hybrid coating. This response is similar to that of a hydrogel, which absorbs water through hydrogen bonding and increases in volume without dissolving in aqueous solutions. The thermal treatment of the film induces a hydrophilicity gradient along the out-of-plane direction of the film, generating preferential swelling of the bottom part of the layer when water is absorbed. This generates a mechanical strain because the surface of the film, which is more condensed and less hydrophilic, is in compression while the bottom part is in tension (Figure 2). The two elastic forces act in opposing directions, leading to the bending momentum that detaches the film from the substrate and produces the self-rolling. The Fourier transformed infrared spectroscopy (FTIR) analysis in the attenuated total reflectance configuration confirmed that the top and bottom parts of the hybrid film have different degrees of condensation, supporting the proposed model of formation (Supplementary Information 6). Surface oxidation treatment by UV-induced ozone 


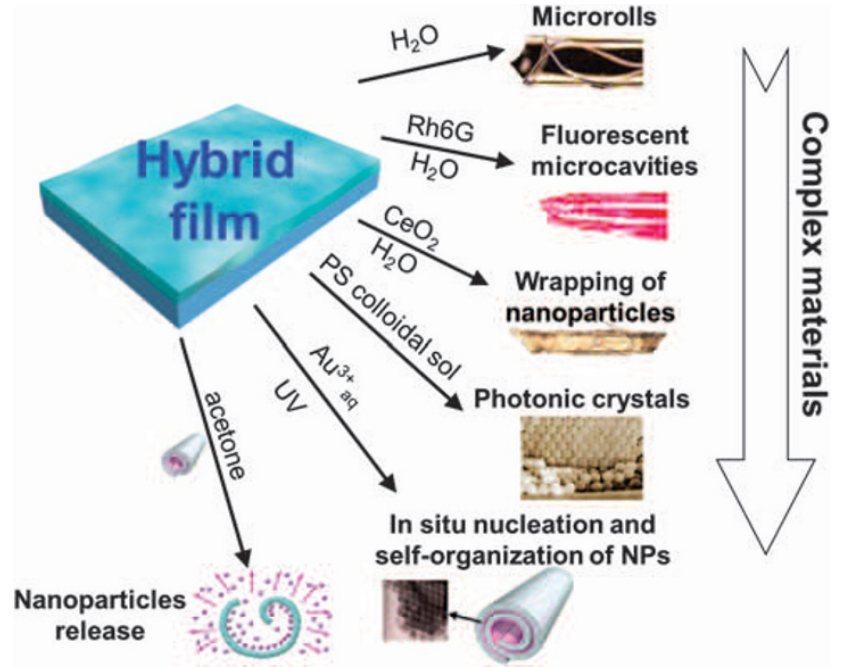

Figure 9 Rolling-up as a multi-functional tool for fabricating complex materials. This drawing reproduces the wide variety of functional materials prepared through self-rolling processing. Microrolls doped with dye molecules, such as Rh6G, can be used as fluorescent microcavities, exploiting the soft chemistry route for film formation. If a colloidal solution is used to start the rolling-up, microrolls can incorporate nanoparticles. When polystyrene microparticles are used instead of nanoparticles, they self-organize into a 3D photonic crystal among the roll coils. Finally, if the gold nanoparticle precursor is added to water before rolling and the microrolls are exposed to UV light after their formation, an ordered array of gold nanoparticles is obtained inside the rolls.

or oxygen plasma is also an effective way to induce scrolling, which confirms that preferential surface condensation activates the mechanical instability (Supplementary Information 7).

The process developed here for the fabrication of hybrid microrolls has an intrinsic advantage in that the overall scrolling of the coating layer is activated by water. This suggests that water could also be used as a solvent for specific molecules or even nano- and microparticles that would remain entrapped at the interface of adjacent roll walls at the end of the process. Different combinations of functional rolls and smart interfaces can be designed and fabricated. In particular, if the nanoparticles are formed in situ at the interface of the two adjacent hybrid layers of the roll, they are in a very peculiar environment, in the sense that it is closed but is still flexible enough to allow for some structural rearrangements of the nanoparticles. A difficult challenge in the synthesis of nanoparticles is in controlling not only the dimension and shape but also the spatial arrangement, which is quite difficult to achieve via self-assembly, especially in 3D. We have observed that in situ formation of gold nanoparticles leads to self-ordering of the nanoparticles at the interface. This phenomenon can be explained by the fact that at the interface between two walls, the gold ions have sufficient mobility to facilitate the nucleation and growth of gold nanoparticles, and the two adjacent layers force the particles to organize into an ordered array at the interface (Figure 6a).

As we have seen, particles with a specific dimension and shape synthesized ex situ can also be entrapped at the roll interface during strain-driven scrolling. The particles form a layer that can be used for different functional applications in several fields, such as catalysis and photonics. At the end of the process incorporating monodisperse PS nanoparticles, opalescent rolls are formed. The opalescence is caused by the organization of the nanoparticles into large domains of photonic crystals at the heterogeneous interface (Figure 6b). The constrained but flexible environment of the roll interfaces also works effectively in this case to create 3D self-ordering. There are multiple advantages of fabricating 3D arrays of nanoparticles located at the roll interface. Different functional molecules can be absorbed by capillarity at the interface, creating contact with the nanoparticles, or can even be introduced in a one-pot step during the scrolling process. Therefore, the interface can have several applications and can easily be used as a sensing layer. The structure of microrolls with gold nanoparticles at the interface makes them able to absorb a liquid at the interface by capillarity. Rh6G and gold nanoparticles coentrapped microrolls exhibited a SERS signal from Rh6G (Figure 8b). This indicates that microrolls can be used as simple and versatile SERS substrates.

Another particular property is that the rolls can be easily opened by acetone. This property can be used for the release of the micro and nanoparticles entrapped at the interface (Supplementary Information 8 and Video). The microroll processing allows complex self-assembly and behavior (Figure 9), including scrolling, organization at the interface and controlled release.

\section{CONCLUSION}

The hybrid organic-inorganic system developed here has attractive properties, and its overall preparation process offers several advantages: one-step-one-layer fabrication, the ability to functionalize walls, and the ability to form self-organize arrays of particles with different sizes and compositions. The extreme flexibility of this fabrication route allows the synthesis of engineered environmentally responsive interfaces combining functional molecules, nanoparticles and microparticles.

\section{ACKNOWLEDGEMENTS}

We acknowledge partial financial support from Grant-in-Aids from the Ministry of Education, Culture, Sports, Science and Technology (MEXT), administered by the Japan Society for the Promotion of Science (JSPS) (No. 22655071, No. 22360276, No. 23656401).

1 Mei, Y., Huang, G., Solovev, A. A., Bermúdez Ureňa, S., Mönch, I., Ding, F., Reindl, T., Fu, K. Y., Chu, P. K. \& Schmidt, O. G. Versatile approach for integrative and functionalized tubes by strain engineering of nanomembranes on polymers. Adv. Mater. 20, 4085-4090 (2008).

2 Zang, J., Huang, M. \& Feng Liu, F. Mechanism for nanotube formation from selfbending nanofilms driven by atomic-scale surface-stress imbalance. Phys. Rev. Lett. 98, 146102-1-4 (2007).

3 Luchnikov, V., Kumar, K. \& Stamm, M. Toroidal hollow-core microcavities produced by self-rolling of strained polymer bilayer films. J. Micromech. Microeng. 18, 3504135046 (2008).

4 Luchnikov, V., Sydorenko, O. \& Stamm, M. Self-rolled polymer and composite/polymer metal micro- and nanotubes with patterned inner walls. Adv. Mater. 17, 1177-1182 (2005)

5 Takahashi, M., Inoue, M., Ihara, R., Yoko, T., Nemoto, T., Isoda, S., Malfatti, L., Costacurta, S. \& Innocenzi, P. Photo-fabrication of titania hybrid films with tunable hierarchical structures and stimuli responsive properties. Adv. Mater. 22, 3303-3306 (2010)

6 Nastauschev, Y. V., Prinz, V. Y. \& Svitasheva, S. N. A technique for fabricating Au/Ti micro- and nanotubes. Nanotechnology 16, 908-913 (2005).

7 Songmuang, R., Denke, C. H. \& Schmidt, O. G. Rolled-up micro- and nanotubes from single-material thin films. Appl. Phys. Lett. 89, 223109-223111 (2006).

8 Schmidt, O. G. \& Eberl, K. Nanotechnology: thin solid films roll up into nanotubes. Nature 410, 168 (2001).

9 Prinz, V. Y., Seleznev, V. A., Gutakovsky, A. K., Chehovskij, A. V., Preobrazhenskii, V. V., Putyato, M. A. \& Gavrilova, T. A. Free-standing and overgrown INGaAs/GaSa nanotubes, nanohelices and their arrays. Physica E 6, 828-831 (2000).

10 Prinz, A. Y. \& Prinz, V. Y. Application of semiconductor micro- and nanotubes in biology. Surf. Sci. 532-535, 911-915 (2003).

11 Luchnikov, V., Ionov, L. \& Stamm, M. Self-rolled polymer tubes: novel tools for microfluidics, microbiology, and drug-delivery systems. Macrom. Rapid Commun. 32, 1943-1952 (2011) 
12 Kalaitzidou, K. \& Crosby, A. J. Adaptive polymer particles. Appl. Phys. Lett. 93, 041910-041913 (2008).

13 Kumar, K., Nandan, B., Luchnikov, V., Simon, F., Vyalikh, A., Scheler, U. \& Stamm, M. A Novel approach for the fabrication of silica and silica/metal hybrid microtubes. Chem. Mater. 21, 4282-4287 (2009).

14 Prinz, A. V., Prinz, V. Y. \& Seleznev, V. A. Semiconductor micro- and nanoneedles for microinjections and ink-jet printing. Microel. Eng. 67-68, 782-788 (2003).

15 Holmes, D. P., Rochè, M., Sinha, T. \& Stone, A. Bending and twisting of soft materials by non-homogeneous swelling. Soft Matter 7, 5188-5193 (2011).

16 Simpson, B., Nunnery, G., Tannenbaum, R. \& Kalaitzodou, K. Capture/release ability of thermo-responsive polymer particles. J. Mater. Chem. 20, 3496-3501 (2010).

17 Stoychev, G., Puretskiy, N. \& Ionov, L. Self-folding all-polymer thermoresponsive microcapsules. Soft Matter 7, 3277-3279 (2011).

18 Innocenzi, P., Kidchob, T. \& Yoko, T. Hybrid organic-inorganic sol-gel materials based on epoxy-amine systems. J. Sol-Gel Sci. Technol. 35, 225-235 (2005).

19 Innocenzi, P., Malfatti, L., Kidchob, T., Aiello, D. \& Aiello, R. Aggregation states of rhodamine G6 in mesostructured silica films. J. Phys. Chem. C 112, 16225-16230 (2008).
20 Takahashi, M., Suzuki, M., Miyagawa, Y., Ihara, R., Tokuda, Y., Yoko, T., Nemoto, T. \& Isoda, S. Photo-curable organically modified silicate-phosphate alternating copolymer for photonics applications. J. Sol-Gel Sci. Technol. 54, 319-324 (2010).

21 Takahashi, M., Figus, C., Kidchob, T., Enzo, S., Casula, M., Valentini, M. \& Innocenzi, P. Self-organized nanocrystalline organosilicates in organic-inorganic hybrid films. Adv. Mater. 21, 1732-1736 (2009).

22 Innocenzi, P., Figus, C., Kidchob, T. \& Takahashi, M. Crystallization in hybrid organicinorganic materials through self-organization from 3-glycidoxypropyltrimethoxysilane. J. Ceram. Soc. Jpn. 119, 387-392 (2011).

(c) This work is licensed under the Creative Commons Attribution-NonCommercial-Share Alike 3.0 Unported License. To view a copy of this license, visit http://creativecommons. org/licenses/by-nc-sa/3.0/

Supplementary Information accompanies the paper on the NPG Asia Materials website (http://www.nature.com/am) 